\title{
Essential Self-Adjointness of the Schrödinger Operators with Magnetic Fields Diverging at Infinity
}

Dedicated to Professor Teruo IKEBE on his sixtieth birthday

By

\section{Akira IwATSUKA*}

\section{§1. Introduction}

In the present paper we shall consider the Schrödinger operators with magnetic fields:

$$
L=-\sum_{j=1}^{n}\left(\partial_{j}-i b_{j}\right)^{2}+V,
$$

where $\partial_{j}=\partial / \partial x_{j}, i=\sqrt{-1}$, and $b_{j}$ and $V$ are the operators of multiplication by real-valued functions on $\boldsymbol{R}^{n}, b_{j}(x)$ and $V(x)$, respectively. $V$ and $\boldsymbol{b}=\left(b_{1}, \cdots, b_{n}\right)$ are called a scalar potential and a (magnetic) vector potential, respectively, and the corresponding magnetic field is the skew symmetric matrix-valued function $B \equiv \operatorname{curl} \boldsymbol{b}$ with $(j, k)$ components

$$
B_{j k}=\partial_{j} b_{k}-\partial_{k} b_{j} \quad \text { for } \quad j, k=1, \cdots, n .
$$

We are concerned with the following property:

(Ess) the restriction $L_{0}$ of $L$ with domain $\mathscr{D}\left(L_{0}\right)=C_{0}^{\infty}\left(\boldsymbol{R}^{n}\right)$ (the set of all $C^{\infty}$ functions on $\boldsymbol{R}^{n}$ with compact support) is essentially self-adjoint as an operator in the Hilbert space $L^{2}\left(\boldsymbol{R}^{n}\right)$.

Sufficient conditions for (Ess) have been investigated extensively by many authors. In the case where $V \geq 0$, Leinfelder and Simader [5] showed that the condition

(P.1) $\boldsymbol{b} \in L_{\mathrm{loc}}^{4}\left(\boldsymbol{R}^{n}\right)^{n}, \operatorname{div} \boldsymbol{b} \in L_{\mathrm{loc}}^{2}\left(\boldsymbol{R}^{n}\right), 0 \leq V \in L_{\mathrm{loc}}^{2}\left(\boldsymbol{R}^{n}\right)$,

Communicated by S. Matsuura, December 18, 1989.

*Department of Mathematics, Kyoto University, Sakyo-ku, Kyoto 606, Japan. 
is sufficient for (Ess), which is decisive as to the assumption on the local singularity of the potentials in the sense that $(\mathbb{P} .1)$ is minimal to assure that $\mathbb{L}_{0}$ defines an operator from $C_{0}^{\infty}\left(\mathbb{R}^{n}\right)$ to $L^{2}\left(\mathbb{R}^{n}\right)$, for we have

$$
\mathbb{L}=-\Delta+2 i b \cdot \nabla+i \operatorname{div} b+|\vec{b}|^{2}+V \text {. }
$$

As to the assumption on the behavior at infinity of the potentials, the result given by Ikebe and Kato [2] is fundamental ([2] allows some local singularities of the potentials; for simplicity, we assume they are $C^{\infty}$ ):

(P.2) there exists a positive non-decreasing function $Q(r)(r \geq 0)$ such that $V(x) \geq-Q(|x|)$ for $x \in \mathbb{R}^{n}\left(|x|=\sqrt{x_{1}^{2}+\cdots+x_{n}^{2}}, x=\left(x_{1}, \cdots, x_{n}\right)\right)$ and $\int^{\infty} Q(r)^{-1 / 2} d r=\infty$

is sufficient for (Ess), which is, roughly speaking, almost necessary as well in the one dimensional case, if one requires a suitable condition on the decay rate at infinity of $V^{\prime}$ and $V^{\prime \prime}$ (see [6, Th.X.9]). Eastham, Evans and Mcleod [1] showed that an estimate of this type is needed only on a sequence of shell-like regions surrounding the origin: for example, the condition

(P.3) there exist a sequence of non-overlapping annular regions $A_{m}=\{x \in$ $\left.\mathbb{R}^{n}\left|a_{m} \leq\right| x \mid \leq b_{m}\right\}$ and a sequence of positive numbers $v_{m}$ such that $\left(b_{m}-a_{m}\right)^{2} v_{m} \geq K, V(x) \geq-k v_{m}^{2}\left(b_{m}-a_{m}\right)^{2}$ for $x \in A_{m}$ and $\sum v_{m}^{-1}=\infty$, where $K$ and $k$ are positive constants independent of $m$

is sufficient for (Ess). Note that these conditions concern the growth rate at infinity of the negative part $V_{-} \equiv \max (0,-V)$ of the scalar potential $V$. As for the magnetic potential, no conditions other than its local regularity are required in these works and, as far as we know, this also seems to be the case with the results so far known.

Our purpose in the present paper is to show that the condition on the growth rate at $\infty$ of $V_{-}$can be relaxed in the presence of the magnetic field $B$ (rather than the magnetic potential $\bar{b}$ because of gauge invariance: see the remark after Theorem 1.1 below). We restrict ourselves to the case of smooth potentials for simplicity, though local singularities might be included: we assume

(H.1) $\quad V(x)$ and $b_{j}(x)$ are real-valued $C^{\infty}$ functions on $\mathbb{R}^{n}$.

Define the magnitude of the skew symmetric matrix $B(x)$ by

$$
|B(x)|=\left\{\sum_{j<k} B_{j k}(x)^{2}\right\}^{1 / 2} .
$$


Our main theorem is the following

Theorem 1.1. Let the assumption (H.1) hold. Suppose that there exists a continuous non-decreasing function $Q(r)>0$ for $r \in[0, \infty)$ such that

$$
\begin{aligned}
& V(x)+|B(x)| \geq-Q(|x|) \quad \text { for } \quad x \in \mathbb{R}^{n}, \\
& \left\{\frac{\left|\partial^{\alpha} B_{j k}(x)\right|}{|B(x)|+1}\right\}^{3-|\alpha|} \leq Q(|x|) \\
& \text { for } \quad x \in \mathbb{R}^{n}, j, k=1, \cdots, n,|\alpha|=1,2, \\
& \quad \int^{\infty} Q(r)^{-1 / 2} d r=\infty,
\end{aligned}
$$

where $\alpha$ are multi-indices $\left(\alpha_{1}, \cdots, \alpha_{n}\right), \partial^{\alpha}=\partial_{1}^{\alpha} \cdots \partial_{n}^{\alpha_{n}}$ and $|\alpha|=\alpha_{1}+\cdots+\alpha_{n}$. Then (Ess) holds.

Remark. It is known as gauge invariance that all $L=L(b)$ with common $B=$ curl $\boldsymbol{b}$ are unitarily equivalent to each other; it follows that the property (Ess) with $V$ fixed depends only on curl $\boldsymbol{b}$. If $\boldsymbol{b}$ is smooth as in our case, then this can be shown quite simply (there are subtleties, however, if $\boldsymbol{b}$ is not smooth; see [4, Theorem 1.3]). In fact, if $\boldsymbol{b}^{\prime}$ is another $C^{\infty}$ vector potential with curl $\boldsymbol{b}^{\prime}=\operatorname{curl} \boldsymbol{b}$, then there exists a real-valued $C^{\infty}$ function $g$ on $\boldsymbol{R}^{n}$ such that $\nabla g=\boldsymbol{b}^{\prime}-\boldsymbol{b}$, which gives $\left(\partial_{j}-i b_{j}^{\prime}\right)=e^{i g}\left(\partial_{j}-i b_{j}\right) e^{-i g}$ and thus $L\left(\boldsymbol{b}^{\prime}\right)=$ $e^{i g} L(b) e^{-i g}$ (gauge invariance); hence we have the equivalence of the essential self-adjointness of $\left.L\left(\boldsymbol{b}^{\prime}\right)\right|_{C_{0}^{\infty}\left(\boldsymbol{R}^{n}\right)}$ and that of $\left.L(\boldsymbol{b})\right|_{c_{0}^{\infty}\left(\boldsymbol{R}^{n}\right)}$ by noting that $e^{i g}$, the operator of multiplication by the $C^{\infty}$ function $e^{i g(x)}$ with modulus 1 , is unitary in $L^{2}\left(\boldsymbol{R}^{n}\right)$ and leaves $C_{0}^{\infty}\left(\boldsymbol{R}^{n}\right)$ invariant.

In view of (1.4) in the above theorem $V_{-}(x)$ is allowed to grow as fast as $|B(x)|+Q(|x|)$, where $Q$ is a function satisfying (1.6), which is the same condition as in (P.2) and by which $Q$ should not grow faster than $r^{\delta}(\delta>2)$. Hence, in the case where $|B(x)|$ grows sufficiently fast, say, at a rate comparable to $|x|^{\delta}(\delta>2)$, Theorem 1.1 gives a wider class of potentials satisfying (Ess) than those given in [2] or [1].

A quantum mechanical interpretation of essential self-adjointness is that the uniqueness of a self-adjoint realization means the uniqueness of the dynamics of the quantum mechanical particle (see, e.g., [1]). If the particle reaches infinity in a finite time, some boundary condition at infinity should be imposed so as to determine a reflection law, in which case $L_{0}$ is not essentially self-adjoint. Thus Theorem 1.1 can be interpreted as follows: the presence of a strong magnetic 
field can prevent the particle from going to infinity in a finite time even though the scalar potential is highly repulsive so that the particle would go to infinity in a finite time if the magnetic field were absent.

\section{§2. General Theorems}

In this section, we are going to state two more theorems, from which Theorem 1.1 can be derived.

First, we give a more general sufficient condition for (Ess).

Theorem 2.1. Let the assumption (H.1) hold. Suppose that there exist a continuous non-decreasing function $Q(r)>0$ for $r \in[0, \infty)$ and an $n \times n$ skew symmetric matrix valued $C^{\infty}$ function $\beta(x)=\left(\beta_{j k}(x)\right)\left(\beta_{j k}\right.$ is real, $\left.\beta_{j k}=-\beta_{k j}\right)$ on $\mathbb{R}^{n}$ such that

$$
\begin{gathered}
|\beta(x)| \leq 1 \quad \text { for } \quad x \in \mathbb{R}^{n}, \\
V(x)+\sum_{j<k} \beta_{j k}(x) B_{j k}(x) \geq-Q(|x|) \quad \text { for } \quad x \in \mathbb{R}^{n}, \\
|R(x)| \leq Q(|x|) \quad \text { for } \quad x \in \mathbb{R}^{n} \text { where } \\
R=-\frac{1}{2} \sum_{j, k, m}\left\{\left(\partial_{j} \beta_{j k}\right)\left(\partial_{m} \beta_{m k}\right)+\beta_{j k}\left(\partial_{j} \partial_{m} \beta_{m k}\right)\right\}+\frac{1}{4} \sum_{j}\left(\sum_{k} \partial_{k} \beta_{k j}\right)^{2}, \\
\int^{\infty} Q(r)^{-1 / 2} d r=\infty .
\end{gathered}
$$

Then (Ess) holds.

Note that the magnitude $|\beta|$ of skew symmetric matrix $\beta$ in (2.1) in the above theorem is defined by

$$
|\beta| \equiv\left\{\sum_{j<k} \beta_{j k}^{2}\right\}^{1 / 2}
$$

as in (1.3).

From this theorem it is known that the condition (1.5) in Theorem 1.1 is rather technical: By putting $\beta$ constant in this theorem, e.g., $\beta_{12}=1, \beta_{21}=-1$, $\beta_{j k}=0$ otherwise, we know that the condition (1.5) can be omitted in Theorem 1.1 if (1.4) is replaced by $V(x)+B_{12}(x) \geq-Q(|x|)$. We can also obtain Theorem 1.1 itself as a particular case of this theorem by putting approximately $\beta_{j k}=$ $B_{j k} /|B|$, as we shall see later in the proof of Theorem 1.1. Theorem 2.1 can be obtained, in turn, as a corollary to a more general theorem given below.

Second, we shall give a theorem concerning a second-order elliptic operator of the form 


$$
T=\sum_{j, k=1}^{n}\left(i \partial_{j}+\bar{f}_{j}\right) G_{j k}\left(i \partial_{k}+f_{k}\right)+W,
$$

where $G_{j k}, f_{j}, W$ and $\bar{f}_{j}$ are operators of multiplication by functions on $\boldsymbol{R}^{n}$, $G_{j k}(x), f_{j}(x), W(x)$ and $\overline{f_{j}(x)}$ (the complex conjugate of $f_{j}(x)$ ), respectively. Note that many authors as [2], [3], or [1] treat operators of the form (2.6), rather than simply of the form (1.1), with real-valued $G_{j k}$ and $f_{j}$. We assume

(H.2) $G_{j k}(x), f_{j}(x)$ are $C^{\infty}$ complex-valued functions on $\boldsymbol{R}^{n}, W(x)$ is a $C^{\infty}$ realvalued function on $\boldsymbol{R}^{n}$ and $G_{j k}(x)=\overline{G_{k j}(x)}$ for $x \in \boldsymbol{R}^{n}$ and $j, k=1, \cdots, n$, and define a symmetric operator $T_{0}$ in $L^{2}\left(\boldsymbol{R}^{n}\right)$ by

$$
\left\{\begin{array}{l}
\mathscr{D}\left(T_{0}\right)=C_{0}^{\infty}\left(\boldsymbol{R}^{n}\right) \\
T_{0} u=T u \quad \text { for } \quad u \in \mathscr{D}\left(T_{0}\right) .
\end{array}\right.
$$

Although $G_{j k}$ are apparently complex-valued in (2.6), it is verified by direct calculation, as is shown in (2.12) below, that the coefficients of the second order terms of $T$ are given in terms of the real part of $G_{j k}$. We further assume the following condition, which is, therefore, nothing but the ellipticity condition on $T$ :

(H.3) The symmetric matrix $\left(\alpha_{j k}(x)\right)$ is positive-definite at each point $x \in \boldsymbol{R}^{n}$ where $\alpha_{j k}(x)=\operatorname{Re} G_{j k}(x)$ (Re means the real part).

We define $\alpha^{*}(r)$ for $r>0$ by

$$
\alpha^{*}(r)=\max _{|x|=r}\left\{\text { the greatest eigenvalue of }\left(\alpha_{j k}(x)\right)\right\} .
$$

Our last theorem is the following

Theorem 2.2. Let the assumptions (H.2) and (H.3) hold. Suppose that the Hermitian matrix $\left(G_{j k}(x)\right)$ is nonnegative-definite, i.e.,

$$
\left(G_{j k}(x)\right) \geq 0 \quad \text { for } \quad x \in \boldsymbol{R}^{n} .
$$

Moreover, suppose that there exist a continuous non-decreasing function $M(r)$ $>0$ for $r \in[0, \infty)$ such that

$$
\begin{gathered}
W(x) \geq-M(|x|) \quad \text { for } \quad x \in \boldsymbol{R}^{n}, \\
\int^{\infty}\left\{\alpha^{*}(r) M(r)\right\}^{-1 / 2} d r=\infty .
\end{gathered}
$$

Then $T_{0}$ is essentially self-adjoint in $L^{2}\left(\boldsymbol{R}^{n}\right)$.

Theorem 2.1 is obtained from Theorem 2.2 by taking $\left(G_{j k}\right) \equiv I+i \beta$ ( $I$ is 
the $n \times n$ identity matrix) with a suitable choice of $f_{j}$ : as we shall see later, $L$ then equals $T$ with $W=V+\sum_{j<k} \beta_{j k} B_{j k}-R$. [2] or [3] have given results quite similar to Theorem 2.2 concerning operators of the form (2.6) with real-valued $G_{j k}$ and $f_{j}$, and Theorem 2.2 can be proved in the same manner as theirs with little modification. We shall give, however, a complete proof of Theorem 2.2 for the sake of self-containedness.

Here we carry out a calculation for the later use in the proof of Theorem 2.1, as well as to show that (H.3), under the assumption (H.2), is the ellipticity condition on $T$.

Put

$$
\left\{\begin{array}{c}
f_{j}=b_{j}+e_{j}, \\
\Pi_{j}=i \partial_{j}+b_{j}
\end{array}\right.
$$

where $b_{j}$ are real-valued $\mathbb{C}^{\infty}$ functions on $\mathbb{R}^{n}$. Then we claim

$$
\begin{aligned}
T= & \sum_{j, k} \Pi_{j}\left(\operatorname{Re} G_{j k}\right) \Pi_{k}+\sum_{j, k}\left\{2 \operatorname{Re}\left(\overline{e_{j}} G_{j k}\right)-\partial_{j}\left(\operatorname{Im} G_{j k}\right)\right\} \Pi_{k} \\
& -\sum_{j<k}\left(\operatorname{Im} G_{j k}\right) B_{j k}+i \sum_{j, k} \partial_{j}\left(G_{j k} e_{k}\right)+\sum_{j, k} G_{j k} \overline{e_{j}} e_{k}+W
\end{aligned}
$$

where $\left(B_{j k}\right)$ is the magnetic field given by (1.2). By this equality, it is known that the coefficient of the second order term $\partial_{j} \partial_{k}$ in $T$ is $-\operatorname{Re} G_{j k}=-\alpha_{j k}$.

To show (2.12), first we have from (2.6),

$$
\begin{aligned}
T & =\sum_{j, k}\left(\Pi_{j}+\overline{e_{j}}\right) G_{j k}\left(\Pi_{k}+e_{k}\right)+W \\
& =\sum_{j, k} \Pi_{j} G_{j k} \Pi_{k}+\sum_{j, k} \overline{e_{j}} G_{j k} \Pi_{k}+\sum_{j, k} \Pi_{j} G_{j k} e_{k}+\sum_{j, k} \overline{e_{j}} G_{j k} e_{k}+W .
\end{aligned}
$$

For the first term on the right we obtain

$$
\begin{aligned}
& \sum_{j, k} \Pi_{j} G_{j k} \Pi_{k} \\
= & \sum_{j_{\diamond} k} \Pi_{j}\left(\operatorname{Re} G_{j k}\right) \Pi_{k}-\sum_{j, k}\left(\partial_{j}\left(\operatorname{Im} G_{j k}\right)\right) \Pi_{k}-\sum_{j<k}\left(\operatorname{Im} G_{j k}\right) B_{j k},
\end{aligned}
$$

because we have

$$
i \sum_{j, k} \Pi_{j}\left(\operatorname{Im} G_{j k}\right) \Pi_{k}=i \sum_{j, k}\left(\operatorname{Im} G_{j k}\right) \Pi_{j} \Pi_{k}-\sum_{j, k}\left\{\partial_{j}\left(\operatorname{Im} G_{j k}\right)\right\} \Pi_{k}
$$

and because we further obtain by using $G_{j k}=\overline{G_{k j}}$ and the commutation relation $\Pi_{j} \Pi_{k}-\Pi_{k} \Pi_{j}=i B_{j k}$,

$$
\begin{aligned}
i \sum_{j, k}\left(\operatorname{Im} G_{j k}\right) \Pi_{j} \Pi_{k} & =i \sum_{j<k}\left(\operatorname{Im} G_{j k}\right)\left\{\Pi_{j} \Pi_{k}-\Pi_{k} \Pi_{j}\right\} \\
& =-\sum_{j<k}\left(\operatorname{Im} G_{j k}\right) B_{j k} .
\end{aligned}
$$


Moreover, we have by using $G_{j k}=\overline{G_{k j}}$ for the third term on the right of (2.13),

$$
\begin{aligned}
\sum_{j, k} \Pi_{j} G_{j k} e_{k} & =\sum_{j, k} G_{j k} e_{k} \Pi_{j}+i \sum_{j, k} \partial_{j}\left(G_{j k} e_{k}\right) \\
& =\sum_{j, k} \overline{G_{j k}} e_{j} \Pi_{k}+i \sum_{j, k} \partial_{j}\left(G_{j k} e_{k}\right) .
\end{aligned}
$$

Thus we have (2.12) from (2.13), (2.14) and (2.15).

\section{§ 3. A Priori Estimate}

To prove Theorem 2.2, we shall make use of the following a priori estimate concerning the operator $T$, which holds not only for $C^{\infty}$ functions with compact support but also for all the $C^{\infty}$ functions on $\mathbb{R}^{n}$, and which is very similar to those utilized also by many authors as [7], [2] and [3] for the proof of the essential self-adjointness of $T_{0}$ with real-valued $G_{j k}$ and $f_{j}$.

Lemma 3.1. Under the same assumptions as in Theorem 2.2, there exists a constant $C$ such that

$$
\int_{\boldsymbol{R}^{n}} \frac{\sum_{j k} G_{j k}(x)\left(i \partial_{k}+f_{k}\right) u(x) \overline{\left(i \partial_{j}+f_{j}\right) u(x)}}{M\left(\Psi^{-1}\{\Psi(|x|)+1\}\right)} d x \leq C\left(\|T u\|^{2}+\|u\|^{2}\right)
$$

for all $u \in C^{\infty}\left(\boldsymbol{R}^{n}\right)$, where $\Psi(r)=\int_{0}^{r} \alpha^{*}(t)^{-1 / 2} d t$ and $\|\cdot\|$ denotes the usual norm of $L^{2}\left(\boldsymbol{R}^{n}\right)$.

We need two elementary propositions for the proof of this lemma:

Proposition 3.2. Let $f(r)>0$ for $r \geq r_{0}$ satisfying $\int_{r_{0}}^{\infty} f(r)^{-1} d r=\infty$ and $g(r) \in$ $L^{1}\left(r_{0}, \infty\right)$. Then $\liminf _{r \rightarrow \infty} f(r)|g(r)|=0$.

Proposition 3.3. Let $Q$ be a continuous non-decreasing function on $[0, \infty)$ satisfying $Q(0) \geq 1$ and $F$ a continuous function on $[0, \infty)$ such that $F>0$ and $\int_{0}^{\infty} F(r) d r=\infty$. Then, for any $\delta>0$, there exists a $C^{1}$ function $\rho$ on $[0, \infty)$ such that

$$
\begin{gathered}
0 \leq \rho^{\prime}(r) \leq F(r) \rho(r)^{1+\delta}, \\
Q(r)<\rho(r)<2 Q\left(\Psi^{-1}\{\Psi(r)+1\}\right)
\end{gathered}
$$

for all $r \geq 0$, where $\Psi(r)=\delta \int_{0}^{r} F(t) d t$.

We shall give proofs of these propositions in the last section. 
Proof of Lemma 3.1. Suppose that the assumptions of Theorem 2.2 hold. To prove the lemma, it is clear that it suffices to consider only the case where $M(0) \geq 1$ and $K \equiv\|u\|^{2}+\|T u\|^{2}<\infty$. Then we can apply Proposition 3.3 with $Q(r)=M(r)$, and $F(r)=2 \alpha^{*}(r)^{-1 / 2}$ and $\delta=1 / 2$, since $\alpha^{*}(r)$ defined in (2.7) is positive by (H.3) and continuous by (H.2) and $\int \alpha^{*}(r)^{-1 / 2} d r=\infty$ by (2.10). Therefore, by Proposition 3.3, there exists a function $\rho \in C^{1}([0, \infty))$ such that

$$
\begin{gathered}
0 \leq \rho^{\prime}(r) \leq 2 \alpha^{*}(r)^{-1 / 2} \rho(r)^{3 / 2}, \\
M(r)<\rho(r)<2 M\left(\Psi^{-1}\{\Psi(r)+1\}\right)
\end{gathered}
$$

for all $r \geq 0$, where

$$
\Psi(r)=\delta \int_{0}^{r} F(t) d t=\int_{0}^{r} \alpha^{*}(t)^{-1 / 2} d t
$$

Let $\zeta(x)=\zeta(r) \equiv 1 / \rho(r) \in C^{1}\left(\boldsymbol{R}^{n} \backslash\{0\}\right)$ where $r=|x|, B_{t}=\left\{x \in \boldsymbol{R}^{n}|| x \mid<t\right\}$ and $S_{t}=\left\{x \in \boldsymbol{R}^{n}|| x \mid=t\right\}$. Then we have by Green's formula,

$$
\begin{array}{r}
\int_{B_{t}}\left[\sum_{j, k}\left(i \partial_{j}+\overline{\left.f_{j}\right)}\left\{\zeta G_{j k}\left(i \partial_{k}+f_{k}\right) u\right\}\right] \bar{u} d x\right. \\
=\int_{B_{t}} \zeta\left\{\sum _ { j , k } G _ { j k } ( i \partial _ { k } + f _ { k } ) u \left(\overline{\left.\left.i \partial_{j}+f_{j}\right) u\right\}} d x\right.\right. \\
\quad+i \int_{S_{t}} \zeta\left\{\sum_{j, k} \nu_{j} G_{j k}\left(i \partial_{k}+f_{k}\right) u\right\} \bar{u} d S,
\end{array}
$$

where $d S$ means the Lebesgue measure on $S_{t}$ and $\nu_{j}=\nu_{j}(x)=x_{j} /|x|$. Put the first term on the right of (3.4) $\Phi(t)$, i.e.,

$$
\Phi(t)=\int_{B_{i}} \zeta\left\{\sum_{j, k} G_{j k}\left(i \partial_{k}+f_{k}\right) u \overline{\left(i \partial_{j}+f_{j}\right) u} d x\right.
$$

for $t>0$. Note that the integrand on the right of (3.5) is non-negative by (2.8). Since by (2.6)

$$
\text { 1.h.s. of }(3.4)=\int_{B_{t}} \zeta(T u-W u) \bar{u} d x+i \int_{B_{t}} \zeta^{\prime}\left(\sum_{j, k} \nu_{j} G_{j k}\left(i \partial_{k}+f_{k}\right) u\right) \bar{u} d x
$$

where $\zeta^{\prime}=\zeta^{\prime}(r)=d \zeta(r) / d r$, we have by (3.4),

$$
\begin{aligned}
\Phi(t)= & \int_{B_{t}} \zeta(T u-W u) \bar{u} d x+i \int_{B_{t}} \zeta^{\prime}\left(\sum_{j, k} \nu_{j} G_{j k}\left(i \partial_{k}+f_{k}\right) u\right) \bar{u} d x \\
& -i \int_{S_{b}} \zeta\left\{\sum_{j, k} \nu_{j} G_{j k}\left(i \partial_{k}+f_{k}\right) u\right\} \bar{u} d S \\
= & I+I I+I I I .
\end{aligned}
$$


Then, since $\zeta|u|^{2} \geq 0$, we have by (2.9)

$$
I \leq \int_{B_{t}} \zeta|(T u) \bar{u}| d x+\int_{B_{t}} \zeta(r) M(r)|u|^{2} d x .
$$

Hence we have

$$
I \leq 2\left(\|u\|^{2}+\|T u\|^{2}\right)=2 K,
$$

since $\zeta(r)=1 / \rho(r)<1 / M(r) \leq 1$ by (3.2). On the other hand, by (2.8), the Schwarz inequality for the sesquilinear form $\sum_{j, k} G_{j k} \xi_{k} \bar{\eta}_{j}$ implies the following pointwise estimate

$$
\begin{aligned}
& \left|\sum_{j, k} \nu_{j} G_{j k}\left(i \partial_{k}+f_{k}\right) u\right| \\
\leq & {\left[\sum_{j, k} G_{j k}\left(i \partial_{k}+f_{k}\right) u\left(\overline{\left.i \partial_{j}+f_{j}\right) u}\right]^{1 / 2}\left[\sum_{j, k} G_{j k} \nu_{k} \bar{\nu}_{j}\right]^{1 / 2} .\right.}
\end{aligned}
$$

Hence, since we have

$$
0 \leq \sum_{j, k} G_{j k} \nu_{k} \bar{\nu}_{j}=\sum_{j, k}\left(\operatorname{Re} G_{j k}\right) \nu_{k} \nu_{j} \leq \alpha^{*}(r)
$$

by (2.7) and by the fact that $\nu_{j}$ is real and $\Sigma_{j} \nu_{j}^{2}=1$, we have

$$
\begin{aligned}
& \left|\sum_{j, k} \nu_{j}(x) G_{j k}(x)\left(i \partial_{k}+f_{k}\right) u(x)\right| \\
\leq & \alpha^{*}(|x|)^{1 / 2}\left[\sum_{j, k} G_{j k}(x)\left(i \partial_{k}+f_{k}\right) u(x) \overline{\left(i \partial_{j}+f_{j}\right) u(x)}\right]^{1 / 2},
\end{aligned}
$$

for all $x \in \boldsymbol{R}^{n}$. Therefore we have by (3.8) the following estimate for the second term of the right-hand side of (3.6):

$$
|I I| \leq \int_{B_{t}}\left|\zeta^{\prime}(r)\right| \alpha^{*}(r)^{1 / 2}\left[\sum_{j, k} G_{j k}\left(i \partial_{k}+f_{k}\right) u\left(\overline{\left(\partial_{j}+f_{j}\right)} u\right]^{1 / 2}|u| d x .\right.
$$

Thus, since we have by (3.1)

$$
\begin{aligned}
\left|\zeta^{\prime}(r)\right| & =\left|\frac{d}{d r}\left\{\frac{1}{\rho(r)}\right\}\right|=\left|\frac{1}{\rho(r)^{2}} \frac{d \rho}{d r}\right| \\
& \leq 2 \alpha^{*}(r)^{-1 / 2} \rho(r)^{-1 / 2}=2 \alpha^{*}(r)^{-1 / 2} \zeta(r)^{1 / 2},
\end{aligned}
$$

we have by using the Schwarz inequality and by (3.5)

$$
\begin{aligned}
|I I| & \leq 2 \int_{B_{t}}\left[\zeta \sum _ { j , k } G _ { j k } ( i \partial _ { k } + f _ { k } ) u \left(\overline{\left.\left(\partial_{j}+f_{j}\right) u\right]^{1 / 2}}|u| d x\right.\right. \\
& \leq 2[\Phi(t)]^{1 / 2}\|u\| \leq \frac{1}{2} \Phi(t)+2 K .
\end{aligned}
$$


Similarly, by (3.8), we have the following estimate for the third term of the right-hand side of (3.6):

$$
\begin{aligned}
|I I I| & \leq \int_{S_{t}} \zeta \alpha^{*}(r)^{1 / 2}\left[\sum_{j, k} G_{j k}\left(i \partial_{k}+f_{k}\right) u \overline{\left(i \partial_{j}+f_{j}\right) u}\right]^{1 / 2}|u| d S \\
& =\zeta(t)^{1 / 2} \alpha^{*}(t)^{1 / 2} \int_{S_{t}}\left[\zeta \sum_{j, k} G_{j k}\left(i \partial_{k}+f_{k}\right) u\left(\overline{\left(i \partial_{j}+f_{j}\right) u}\right]^{1 / 2}|u| d S .\right.
\end{aligned}
$$

Thus, since $|\zeta(t)| \leq 1$, we have by using the Schwarz inequality and by (3.5)

$$
|I I I| \leq \alpha^{*}(t)^{1 / 2}\left[\Phi^{\prime}(t)\right]^{1 / 2}\left[\int_{S_{t}}|u|^{2} d S\right]^{1 / 2} .
$$

Therefore we have by (3.6), (3.7), (3.9) and (3.10)

$$
\Phi(t) \leq 4 K+\frac{1}{2} \Phi(t)+\alpha *(t)^{1 / 2}\left[\Phi^{\prime}(t)\right]^{1 / 2}\left[\int_{s_{t}}|u|^{2} d S\right]^{1 / 2},
$$

which implies

$$
\Phi(t) \leq 8 K+2 \alpha^{*}(t)^{1 / 2}\left[\Phi^{\prime}(t)\right]^{1 / 2}\left[\int_{S t}|u|^{2} d S\right]^{1 / 2}
$$

We shall show that (3.11) with $\Phi(t) \geq 0$ and $\Phi^{\prime}(t) \geq 0$ implies

$$
\lim _{t \rightarrow \infty} \Phi(t) \leq 16 K,
$$

which is, by (3.5), equivalent to

$$
\int_{\boldsymbol{R}^{n}} \zeta\left\{\sum_{j, k} G_{j k}\left(i \partial_{k}+f_{k}\right) u \overline{\left(i \partial_{j}+f_{j}\right)} u\right\} d x \leq 16 K .
$$

Then we obtain the required inequality

$$
\int \frac{\sum_{j, k} G_{j k}\left(i \partial_{k}+f_{k}\right) u \overline{\left(i \partial_{j}+f_{j}\right) u}}{M\left(\Psi^{-1}\{\Psi(|x|)+1\}\right)} d x \leq 32 K=32\left(\|T u\|^{2}+\|u\|^{2}\right),
$$

since $\zeta(r)=\rho(r)^{-1} \geq 2^{-1} M\left(\Psi^{-1}\{\Psi(r)+1\}\right)^{-1}$ by (3.2) and $\Psi$ is defined by (3.3).

Therefore it remains to show that (3.11) with $\Phi(t) \geq 0$ and $\Phi^{\prime}(t) \geq 0$ implies (3.12). If $\Phi(t) \equiv 0$ for $t>0$, then clearly (3.12) holds. Thus, since $\Phi(t) \geq 0$, we prove (3.12) assuming $\Phi\left(r_{0}\right)>0$ for some $r_{0}>0$. Since $\Phi^{\prime}(r) \geq 0$ and $\int_{r_{0}}^{A} \frac{\Phi^{\prime}(r)}{\Phi(r)^{2}} d r=\frac{1}{\Phi\left(r_{0}\right)}-\frac{1}{\Phi(A)}$ for all $A \geq r_{0},\left[\Phi^{\prime}(t)\right]^{1 / 2} / \Phi(t) \in L^{2}\left(r_{0}, \infty\right)$. Therefore, since $\left[\int_{s_{t}}|u|^{2} d S\right]^{1 / 2} \in L^{2}\left(r_{0}, \infty\right)$, we have

$$
g(t) \equiv \frac{\left[\Phi^{\prime}(t)\right]^{1 / 2}}{\Phi(t)}\left[\int_{s_{t}}|u|^{2} d S\right]^{1 / 2} \in L^{1}\left(r_{0}, \infty\right)
$$


which enables us to apply Proposition 3.2 with this $g(t)$ and $f(t)=\alpha^{*}(t)^{1 / 2}$ since $\int_{r_{0}}^{\infty} \alpha^{*}(t)^{-1 / 2} d t=\infty$. Thus we obtain by Proposition 3.2

$$
\liminf _{t \rightarrow \infty} \frac{\left[\Phi^{\prime}(t)\right]^{1 / 2}}{\Phi(t)}\left[\int_{s_{t}}|u|^{2} d S\right]^{1 / 2} \alpha^{*}(t)^{1 / 2}=0
$$

Therefore there exists a sequence $\left\{t_{n}\right\}$ such that $t_{n} \rightarrow \infty$ and

$$
\left[\Phi^{\prime}\left(t_{n}\right)\right]^{1 / 2}\left[\int_{s_{t_{n}}}|u|^{2} d S\right]^{1 / 2} \alpha^{*}\left(t_{n}\right)^{1 / 2} \leq \frac{1}{4} \Phi\left(t_{n}\right)
$$

Then we have by (3.11) $\Phi\left(t_{n}\right) \leq 8 K+\frac{1}{2} \Phi\left(t_{n}\right)$. Therefore, since $\Phi(t)$ is nondecreasing, $\lim _{t \rightarrow \infty} \Phi(t)=\lim _{n \rightarrow \infty} \sup \Phi\left(t_{n}\right) \leq 16 K$, which proves (3.12).

\section{§. Proofs of the Theorems}

In this section we shall give proofs of the theorems given in Sections 1 and 2.

Proof of Theorem 2.2. Since $T_{0}$ is a symmetric operator in $L^{2}\left(\boldsymbol{R}^{n}\right)$, the essential self-adjointness of $T_{0}$ is, as is well-known (see, e.g., [6, Theorem X.1]), equivalent to the property that $T_{0}^{*} u= \pm i u$ implies $u \equiv 0$ for $u \in \mathscr{D}\left(T_{0}^{*}\right)$. We only treat the case of $T_{0}^{*} u=i u$, because the case of $T_{0}^{*} u=-i u$ can be proved in the same way.

Suppose that $u \in \mathscr{D}\left(T_{0}^{*}\right)$ and $T_{0}^{*} u=i u$. Then $u \in L^{2}\left(\boldsymbol{R}^{n}\right)$ and $T u=i u$ holds in the distribution sense. Since $T$ is elliptic by (H.3), we have $u \in C^{\infty}\left(\boldsymbol{R}^{n}\right)$ by (H.2). By Green's formula (apply (3.4) with $\zeta \equiv 1$ ), we have

$$
\begin{aligned}
& \int_{B_{t}}(T u-W u) \bar{u} d x \\
= & \int_{B_{t}}\left\{\sum_{j, k} G_{j k}\left(i \partial_{k}+f_{k}\right) u\left(\overline{\left(\partial_{j}+f_{j}\right) u}\right\} d x+i \int_{S_{t}}\left\{\sum_{j, k} \nu_{j} G_{j k}\left(i \partial_{k}+f_{k}\right) u\right\} \bar{u} d S .\right.
\end{aligned}
$$

Since $T u=i u, G_{j k}=\overline{G_{k j}}$ and $W$ is real-valued, by taking the imaginary part of this equality, we have,

$$
\int_{B_{t}}|u|^{2} d x=\operatorname{Im}\left[i \int_{S_{t}}\left\{\sum_{j, k} \nu_{j} G_{j k}\left(i \partial_{k}+f_{k}\right) u\right\} \bar{u} d S\right] .
$$

Therefore, we have by (3.8) and by the Schwarz inequality

$$
\int_{B_{t}}|u|^{2} d x \leq \alpha^{*}(t)^{1 / 2}\left[\int_{S_{t}} \sum_{j, k} G_{j k}\left(i \partial_{k}+f_{k}\right) u \overline{\left(i \partial_{j}+f_{j}\right) u} d S\right]^{1 / 2}\left[\int_{s_{t}}|u|^{2} d S\right]^{1 / 2}
$$


On the other hand, since $T u=i u \in L^{2}\left(\boldsymbol{R}^{n}\right)$, we have by Lemma 3.1,

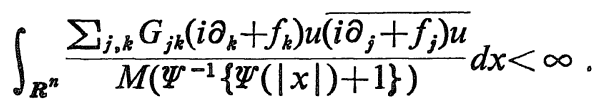

Therefore,

$$
h(t) \equiv\left[\int_{s_{t}} \frac{\sum_{j, k} G_{j k}\left(i \partial_{k}+f_{k}\right) u \overline{\left(i \partial_{j}+f_{j}\right) u}}{M\left(\Psi^{-1}\{\Psi(|x|)+1\}\right)} d S\right]^{1 / 2} \in L^{2}(1, \infty),
$$

which, with $\left[\int_{S_{i}}|u|^{2} d S\right]^{1 / 2} \in L^{2}(1, \infty)$, implies,

$$
g(t) \equiv h(t) \cdot\left[\int_{s_{t}}|u|^{2} d S\right]^{1 / 2} \in L^{1}(1, \infty)
$$

Moreover let $f(t) \equiv\left[\alpha^{*}(t) M\left(\Psi^{-1}\{\Psi(t)+1\}\right)\right]^{1 / 2}$. Then we have by (3.3)

$$
\begin{aligned}
\int_{0}^{\infty} \frac{d t}{f(t)} & =\int_{0}^{\infty} \frac{d \Psi(t)}{M\left(\Psi^{-1}\{\Psi(t)+1\}\right)^{1 / 2}}=\int_{0}^{\infty} \frac{d s}{M\left(\Psi^{-1}\{s+1\}\right)^{1 / 2}} \\
& =\int_{\Psi^{-1}(1)}^{\infty} \frac{d \Psi(t)}{M(t)^{1 / 2}}=\int_{\Psi^{-1}(1)}^{\infty} \frac{d r}{\left[M(r) \alpha^{*}(r)\right]^{1 / 2}},
\end{aligned}
$$

which equals $\infty$ by (2.10). Thus we can apply Proposition 3.2 with these $f$ and $g$ by (4.2) to obtain $\liminf _{t \rightarrow \infty} f(t)|g(t)|=0$. Now, since the right-hand side of (4.1) equals $f(t)|g(t)|$, we have $\int_{R^{n}}|u|^{2} d x=0$, i.e., $u \equiv 0$.

For the proof of Theorem 2.1, we need an elementary proposition:

Proposition 4.1. Let $\beta=\left(\beta_{j k}\right)$ be an $n \times n$ matrix, where $\beta_{j k}$ is real, $\beta_{j k}=$ $-\beta_{k j}$. Then we have

$$
-|\beta| \leq i \beta \leq|\beta|
$$

where $|\beta|$ is given in (2.5).

A proof of this proposition will be given in the last section.

Proof of Theorem 2.1. First, define

$$
G_{j k} \equiv \delta_{j k}+i \beta_{j k}
$$

where $\delta_{j k}$ is the Kronecker delta. Then, since $\beta_{j k}$ is real and $\beta_{j k}=-\beta_{k j}$, $G_{j k}=\overline{G_{k j}}$. Moreover, (2.1) implies (2.8) according to Proposition 4.1. (H.3) is fulfilled and $\alpha^{*}(r) \equiv 1$ because $\alpha_{j k}(x)=\operatorname{Re} G_{j k}(x)=\delta_{j k}$. Second, define 


$$
\left\{\begin{array}{l}
e_{j}=\frac{1}{2} \sum_{k} \partial_{k} \beta_{k j}, \\
f_{j}=b_{j}+e_{j} .
\end{array}\right.
$$

Then we have

$$
\sum_{j}\left\{2 \operatorname{Re}\left(\overline{e_{j}} G_{j k}\right)-\partial_{j}\left(\operatorname{Im} G_{j k}\right)\right\}=2 e_{k}-\sum_{j} \partial_{j} \beta_{j k} \equiv 0 .
$$

Moreover, since $\sum_{j} \partial_{j} e_{j}=\frac{1}{2} \sum_{j, k}\left(\partial_{j} \partial_{k} \beta_{j k}\right) \equiv 0$ and $\sum_{j, k} \beta_{j k} e_{j} e_{k} \equiv 0$ by $\beta_{j k}=$ $-\beta_{k j}$, we have

$$
i \sum_{j, k} \partial_{j}\left(G_{j k} e_{k}\right)+\sum_{j, k} G_{j k} \bar{e}_{j} e_{k}=-\sum_{j, k} \partial_{j}\left(\beta_{j k} e_{k}\right)+\sum_{j} e_{j}^{2},
$$

which is equal to $R$ by (2.3) and (4.4). Therefore, we have by (2.12) and (4.5),

$$
T=\sum_{j} \Pi_{j}^{2}-\sum_{j<k} \beta_{j k} B_{j k}+R+W,
$$

which will coincide with $L$ if we take

$$
W=V+\sum_{j<k} \beta_{j k} B_{j k}-R .
$$

Then we have by (2.2) and (2.3) $W(x) \geq-2 Q(|x|)$, from which, if we further take $M=2 Q$, (2.9) is satisfied. (H.2) is satisfied by these $G_{j k}, f_{j}$ and W. Finally (2.10) is satisfied by (2.4), since $\alpha^{*}(r) \equiv 1$. Now we can apply Theorem 2.2 to prove that the property (Ess) holds, since $T_{0}=L_{0}$ by (4.6).

Proof of Theorem 1.1. Let $\phi(r)$ be a $C^{\infty}$ function on $\boldsymbol{R}$ such that

$$
\begin{aligned}
& \phi(r)=\left\{\begin{array}{lll}
1 & \text { for } & r \leq 1 / 2, \\
1 / r & \text { for } & r \geq 2,
\end{array}\right. \\
& 0<\phi(r) \leq 1 / r \text { for all } r>0 \text {. }
\end{aligned}
$$

And define

$$
\beta_{j k}(x)=\phi(|B(x)|) B_{j k}(x) \quad \text { for } j, k=1, \cdots, n .
$$

Then, by (4.7), (1.2) and (H.1), $\beta_{j k} \in C^{\infty}\left(\boldsymbol{R}^{n}\right)$ is real and satisfies $\beta_{j k}=-\beta_{k j}$. (2.1) holds by (1.3), (2.5) and (4.8). Moreover, according to (4.9) and (4.7), there exists a constant $C$ such that

$$
\sum_{j<k} \beta_{j k} B_{j k}=\phi(|B|)|B|^{2} \geq|B|-C .
$$

Thus, it follows from (1.4) that (2.2) holds with $Q$ replaced by $Q+C$, and hence with $Q$ replaced by $C_{1} Q$ for sufficiently large constant $C_{1}$, since $Q(r) \geq Q(0)>0$ for $r \geq 0$. 
From (4.9) by a direct calculation, we have

$$
\partial_{l} \beta_{j k}=\frac{1}{|B|} \phi^{\prime}(|B|) \sum_{s<t}\left(\partial_{l} B_{s t}\right) B_{s t} B_{j k}+\phi(|B|) \partial_{l} B_{j k},
$$

which with (4.7) shows

$$
\left|\partial_{l} \beta_{j k}(x)\right| \leq\left\{\begin{array}{lll}
C_{2} \frac{1}{|B(x)|} \max _{s, s}\left|\partial_{l} B_{s t}(x)\right| & \text { for } & |B(x)| \geq 1, \\
C_{2} \max _{s, s}\left|\partial_{l} B_{s t}(x)\right| & \text { for } & |B(x)| \leq 1,
\end{array}\right.
$$

for sufficiently large constant $C_{2}$. Therefore we have

$$
\left|\partial_{l} \beta_{j k}(x)\right| \leq \frac{2 C_{2}}{|B(x)|+1} \max _{s, t}\left|\partial_{l} B_{s t}(x)\right|
$$

for all $x \in \boldsymbol{R}^{3}$. Next, we have

$$
\begin{aligned}
& \partial_{m} \partial_{l} \beta_{j k} \\
= & \left\{\frac{\phi^{\prime \prime}(|B|)}{|B|^{2}}-\frac{\phi^{\prime}(|B|)}{|B|^{3}}\right\}\left[\sum_{\mu^{\prime \nu}}\left(\partial_{m} B_{\mu \nu}\right) B_{\mu \nu}\right]\left[\sum_{s<t}\left(\partial_{l} B_{s t}\right) B_{s t}\right] B_{j k} \\
& +\frac{\phi^{\prime}(|B|)}{|B|} \sum_{s<t}\left\{\left(\partial_{m} \partial_{l} B_{s t}\right) B_{s t} B_{j k}+\left(\partial_{l} B_{s t}\right)\left(\partial_{m} B_{s t}\right) B_{j k}+\left(\partial_{l} B_{s t}\right) B_{s t}\left(\partial_{m} B_{j k}\right)\right\} \\
& +\frac{\phi^{\prime}(|B|)}{|B|} \sum_{s<t}\left(\partial_{m} B_{s t}\right) B_{s t}\left(\partial_{l} B_{j k}\right)+\phi(|B|) \partial_{m} \partial_{l} B_{j k},
\end{aligned}
$$

from which, similarly for (4.10), we obtain with some constant $C_{3}$ chosen sufficiently large,

$$
\left|\partial_{m} \partial_{l} \beta_{j k}(x)\right| \leq C_{3}\left\{\frac{\max _{i, s, t}\left|\partial_{i} B_{s t}(x)\right|}{|B(x)|+1}\right\}^{2}+C_{3} \frac{\max _{s, t}\left|\partial_{m} \partial_{l} B_{s t}(x)\right|}{|B(x)|+1}
$$

for all $x \in \mathbb{R}^{n}$. In view of (4.10) and (4.11), it follows from (1.5) that (2.3) is fulfilled if $Q$ is replaced by $C_{4} Q$ with sufficiently large constant $C_{4}$.

Consequently, we have shown that the assumptions of Theorem 2.1 are satisfied, provided that the assumptions of Theorem 1.1 are satisfied, $\beta$ is taken as in (4.9) and $Q$ is replaced by $C_{5} Q$ with suitable constant $C_{5}\left(=\max \left\{C_{1}, C_{4}\right\}\right.$ in the above argument), since (2.4) then follows from (1.6). Thus we can prove Theorem 1.1 by applying Theorem 2.1 .

\section{$\S 5 . \quad$ Proofs of the Propositions}

In this section, we shall give proofs of the propositions; that of Proposition 
3.3 will be given in the last place.

Proof of Proposition 3.2. Suppose that $\liminf _{r \rightarrow \infty} f(r)|g(r)| \neq 0$. Then, since $f(r)>0$, it follows that there exist $\varepsilon>0$ and $r_{1} \geq r_{0}$ such that $f(r)|g(r)| \geq \varepsilon$ for all $r \geq r_{1}$. Namely, $|g(r)| \geq \varepsilon f(r)^{-1}$ for $r \geq r_{1}$, which induces a contradiction since $g(r) \in L^{1}\left(r_{0}, \infty\right)$ and $\int_{r_{0}}^{\infty} f(r)^{-1} d r=\infty$. Thus $\liminf _{r \rightarrow \infty} f(r)|g(r)|$ should equal 0.

Proof of Proposition 4.1. Let $u={ }^{t}\left(u_{1}, \cdots, u_{n}\right)$ and $v={ }^{t}\left(v_{1}, \cdots, v_{n}\right)$ be column vectors with $n$ complex components and denote $(u, v)=\sum_{j=1}^{n} u_{j} \overline{v_{j}}$. Then, by the fact that $\beta_{j k}$ are real and by the relation $\beta_{j k}=-\beta_{k j}$, $(i \beta u, u)$ is real and we have

$$
\begin{aligned}
(i \beta u, u) & =i \sum_{j, k=1}^{n} \beta_{j k} u_{k} \overline{u_{j}} \\
& =i \sum_{j<k} \beta_{j k} u_{k} \overline{u_{j}}+i \sum_{j>k} \beta_{j k} u_{k} \overline{u_{j}} \\
& =i \sum_{j<k} \beta_{j k}\left(u_{k} \overline{u_{j}}-u_{j} \overline{u_{k}}\right) .
\end{aligned}
$$

Hence we have by the Schwarz inequality,

$$
|(i \beta u, u)| \leq\left(\sum_{j<k} \beta_{j k}^{2}\right)^{1 / 2}\left(\sum_{j<k}\left|u_{k} \overline{u_{j}}-u_{j} \overline{u_{k}}\right|^{2}\right)^{1 / 2} .
$$

We have by a direct calculation,

$$
\begin{aligned}
\sum_{j<k}\left|u_{k} \overline{u_{j}}-u_{j} \overline{u_{k}}\right|^{2} & =\frac{1}{2} \sum_{j, k}\left|u_{j} \overline{u_{k}}-u_{k} \overline{u_{j}}\right|^{2} \\
& =\frac{1}{2} \sum_{j, k}\left(u_{j} \overline{u_{k}}-u_{k} \overline{u_{j}}\right)\left(u_{j} \overline{u_{k}}-\overline{u_{k}} u_{j}\right) \\
& =\frac{1}{2} \sum_{j, k}\left\{\left|u_{j}\right|^{2}\left|u_{k}\right|^{2}-u_{j}^{2} \overline{u_{k}}-u_{k}^{2} \bar{u}_{j}^{2}+\left|u_{k}\right|^{2}\left|u_{j}\right|^{2}\right\} \\
& =\left\{\sum_{j}\left|u_{j}\right|^{2}\right\}^{2}-\left|\sum_{j} u_{j}^{2}\right|^{2} \leq|(u, u)|^{2} .
\end{aligned}
$$

Therefore, by (5.1), we have $-|\beta|(u, u) \leq(i \beta u, u) \leq|\beta|(u, u)$.

For the proof of Proposition 3.3, we need another proposition:

Proposition 5.1. Let $-\infty<a<b<\infty, h \in L^{1}(a, b)$. Suppose that $G \in$ $C[a, b], G(x)>0$ for all $x \in[a, b], 0 \leq h \leq G$ a.e. on $(a, b)$ and $\int_{a}^{b} h d x<\int_{a}^{b} G d x$. Then, for any $\varepsilon>0$, there exists some $g \in C_{0}(a, b)$ such that $0 \leq g \leq G$ on $[a, b]$, $\int_{a}^{b} g d x=\int_{a}^{b} h d x$ and $\int_{a}^{b}|g-h| d x \leq \varepsilon$, where $C_{0}(a, b)$ is the set of all the conti- 
nuous functions on $[a, b]$ with compact support contained in $(a, b)$.

Proof. We shall write $\int_{a}^{b} f d x=\int f$. First note that the family of functions $\mathcal{G} \equiv\left\{g \in C_{0}(a, b) \mid 0 \leq g \leq G\right.$ on $\left.[a, b]\right\}$ has the properties:

$$
g_{1}, g_{2} \in \mathcal{G} \text { implies } \lambda g_{1}+(1-\lambda) g_{2} \in \mathcal{G} \quad \text { for } \quad 0 \leq \lambda \leq 1 \text {, }
$$

$$
\begin{array}{r}
g_{1}, g_{2} \in \mathcal{G} \text { implies } g_{1} \vee g_{2}, g_{1} \wedge g_{2} \in \mathcal{G}, \\
\sup _{g \in \mathcal{G}} \int g=\int G,
\end{array}
$$

where $g_{1} \vee g_{2}(x) \equiv \max \left\{g_{1}(x), g_{2}(x)\right\}, g_{1} \wedge g_{2}(x) \equiv \min \left\{g_{1}(x), g_{2}(x)\right\}$.

Let $\varepsilon>0$. Choose some $g_{0} \in C_{0}(a, b)$ such that $\int\left|h-g_{0}\right| \leq \varepsilon / 2$. Let $g_{1} \equiv$ $\left(g_{0} \vee 0\right) \wedge G$. Then, since $G$ is continuous and $>0, g_{1} \in \mathcal{G}$. Moreover, $g_{1}$ satisfies $\int\left|h-g_{1}\right| \leq \varepsilon / 2$. In fact, we have

$$
\begin{aligned}
g_{1}-h & =\left(\left(g_{0}-h\right) \vee(-h)\right) \wedge(G-h) \\
& \leq\left(g_{0}-h\right) \vee(-h) \leq\left(g_{0}-h\right) \vee 0 \quad(\text { by }-h \leq 0) .
\end{aligned}
$$

Similarly we have

$$
\begin{aligned}
g_{1}-h & =\left(\left(g_{0}-h\right) \wedge(G-h)\right) \vee(-h) & & (\text { by }-h \leq G-h) \\
& \geq\left(g_{0}-h\right) \wedge(G-h) \geq\left(g_{0}-h\right) \wedge 0 & & (\text { by } G-h \geq 0),
\end{aligned}
$$

and hence $\left|g_{1}-h\right| \leq\left|g_{0}-h\right|$.

First consider the case where $\int g_{1} \leq \int h$. Then, since $\int h<\int G$, we know by using (5.3) and (5.4) that there is some $g_{2} \in G$ such that $g_{1} \leq g_{2}$ and $\int g_{1}<\int h<\int g_{2}<\int G$. Put $g \equiv g_{1}+\lambda\left(g_{2}-g_{1}\right)$ where $\lambda=\int\left(h-g_{1}\right) / \int\left(g_{2}-g_{1}\right)$. Then clearly $\int g=\int h$, and $g \in \mathcal{G}$ by (5.2). Since $\int\left|g-g_{1}\right|=\lambda \int\left|g_{2}-g_{1}\right|=$ $\lambda \int\left(g_{2}-g_{1}\right)=\int\left(h-g_{1}\right) \leq \varepsilon / 2$, we have $\int|g-h| \leq \varepsilon$. Therefore, this $g$ has the required property.

Second, in the case where $\int h \leq \int g_{1}$, take $g \equiv \lambda g_{1}$, where $\lambda=\int h / \int g_{1}(\lambda=0$ if $\left.\int g_{1}=0\right)$. Then it is not difficult to verify that $g$ has the required property, since $\int\left|g_{1}-g\right|=\int\left(g_{1}-g\right) \leq \int\left(g_{1}-h\right) \leq \varepsilon / 2$.

Proof of Proposition 3.3. Write $R(r) \equiv 2 Q\left(\Psi^{-1}\{\Psi(r)+1\}\right)$ and let 


$$
\begin{aligned}
& \psi^{*}(r) \equiv \min _{\delta \geq r}\left(\Psi(t)+\frac{1}{Q(t)^{\delta}}\right) \\
& \psi_{*}(r) \equiv \max _{t \leq r}\left(\Psi(t)+\frac{1}{R(t)^{\delta}}\right)
\end{aligned}
$$

Note that, from these definitions, it follows immediately

$$
\begin{aligned}
& \psi^{*}(r) \leq \Psi(r)+\frac{1}{Q(r)^{\delta}}, \\
& \psi_{*}(r) \geq \Psi(r)+\frac{1}{R(r)^{\delta}}
\end{aligned}
$$

First we claim

$$
\begin{aligned}
& \psi_{*}(r)<\psi^{*}(r) \quad \text { for all } r \geq 0, \\
& 0 \leq \psi^{*}(s)-\psi^{*}(r) \leq \Psi(s)-\Psi(r) \quad \text { for } \quad r \leq s \text {, } \\
& 0 \leq \psi_{*}(s)-\psi_{*}(r) \leq \Psi(s)-\Psi(r) \quad \text { for } \quad r \leq s .
\end{aligned}
$$

In fact, first note that, to show (5.9), it suffices to prove that

$$
\Psi(r)+\frac{1}{R(r)^{\delta}}<\Psi(s)+\frac{1}{Q(s)^{\delta}} \quad \text { for all } \quad r \leq s .
$$

Suppose that this does not hold. Then,

$$
\Psi(r)+\frac{1}{R(r)^{\delta}} \geq \Psi(s)+\frac{1}{Q(s)^{\delta}} \quad \text { for some } \quad r \leq s .
$$

Since $R(r) \geq Q(r) \geq Q(0) \geq 1$, the left hand side of (5.12) is less than $\Psi(r)+1$. Thus by (5.12) $\Psi(r)+1 \geq \Psi(s)$, which means $\Psi^{-1}\{\Psi(r)+1\} \geq s$. Therefore, since $Q$ is non-decreasing, we have $Q\left(\Psi^{-1}\{\Psi(r)+1\}\right) \geq Q(s)$. However, this contradicts (5.12), because $Q\left(\Psi^{-1}\{\Psi(r)+1\}\right)<R(r)$ and $\Psi(r) \leq \Psi(s)$ by $\Psi^{\prime}=$ $\delta F>0$.

Second, by the definitions (5.5) and (5.6), it is clear that $\psi^{*}$ and $\psi_{*}$ are non-decreasing, that is to say, the first inequalities of (5.10) and (5.11) hold. Let us consider the second inequality of (5.10). Take $r^{*} \geq r$ according to (5.5) such that

$$
\psi^{*}(r)=\Psi\left(r^{*}\right)+\frac{1}{Q\left(r^{*}\right)^{\delta}}
$$

Then $\psi^{*}(r)=\psi^{*}\left(r^{*}\right)$ by (5.5). Let $s \geq r$. If $s \leq r^{*}$ then $\psi^{*}(r) \leq \psi^{*}(s) \leq \psi^{*}\left(r^{*}\right)$ $=\psi^{*}(r)$ and hence $\psi^{*}(s)=\psi^{*}(r)$. If $s>r^{*}$ then, by (5.7) and (5.13), 


$$
\begin{aligned}
\psi^{*}(s)-\psi^{*}(r) & \leq \Psi(s)+\frac{1}{Q(s)^{\delta}}-\left(\Psi\left(r^{*}\right)+\frac{1}{Q\left(r^{*}\right)^{\delta}}\right) \\
& \leq \Psi(s)-\Psi\left(r^{*}\right) \leq \Psi(s)-\Psi(r)
\end{aligned}
$$

by the monotonicity of $Q$ and $\Psi$. We have thus (5.10). We can prove the second inequality of (5.11) by a similar argument taking $s_{*} \leq s$ such that

$$
\psi_{*}(s)=\Psi\left(s_{*}\right)+\frac{1}{R\left(s_{*}\right)^{\delta}}:
$$

$\psi_{*}(s)=\psi_{*}\left(s_{*}\right)$ by (5.6). Let $r \leq s$. If $r \geq s_{*}$ then $\psi_{*}(s) \geq \psi_{*}(r) \geq \psi_{*}\left(s_{*}\right)=$ $\psi_{*}(s)$ and hence $\psi_{*}(s)=\psi_{*}(r)$. If $r<s_{*}$ then

$$
\begin{aligned}
\psi_{*}(s)-\psi_{*}(r) & \leq \Psi\left(s_{*}\right)+\frac{1}{R\left(s_{*}\right)^{\delta}}-\left(\Psi(r)+\frac{1}{R(r)^{\delta}}\right) \\
& \leq \Psi\left(s_{*}\right)-\Psi(r) \leq \Psi(s)-\Psi(r) .
\end{aligned}
$$

Thus we have the claims (5.9), (5.10) and (5.11).

By (5.10) and (5.11) it is known that $\psi^{*}$ and $\psi_{*}$ are absolutely continuous and

$$
\begin{array}{ll}
0 \leq \frac{d \psi^{*}}{d r} \leq \Psi^{\prime}=\delta F & \text { a.e. on } \quad(0, \infty), \\
0 \leq \frac{d \psi_{*}}{d r} \leq \Psi^{\prime}=\delta F & \text { a.e. on }(0, \infty)
\end{array}
$$

We shall show that (5.9), (5.14) and (5.15) imply the existence of a function $\tilde{\psi} \in C^{1}[0, \infty)$ such that

$$
\begin{array}{ll}
\psi_{*}(r)<\tilde{\psi}(r)<\psi^{*}(r) & \text { for all } \quad r \in[0, \infty), \\
0 \leq \frac{d \tilde{\psi}(r)}{d r} \leq \delta F(r) & \text { for all } \quad r \in[0, \infty) .
\end{array}
$$

Assuming these properties we can define $\rho$ by

$$
\tilde{\psi}(r)=\Psi(r)+\frac{1}{\rho(r)^{\delta}},
$$

i.e., $\rho(r) \equiv\{\tilde{\psi}(r)-\Psi(r)\}^{-1 / \delta}$. By (5.16) and (5.8) $\rho \in C^{1}[0, \infty)$ and also by (5.16), (5.7) and (5.8) we have

$$
Q(r)<\rho(r)<R(r)
$$

and, by (5.17) 


$$
0 \leq \delta F(r)-\delta \frac{\rho^{\prime}(r)}{\rho(r)^{1+\delta}} \leq \delta F(r)
$$

which is equivalent to

$$
0 \leq \rho^{\prime}(r) \leq F(r) \rho(r)^{1+\delta} \text {. }
$$

Since we have taken $R(r)=2 Q\left(\Psi^{-1}\{\Psi(r)+1\}\right),(5.18)$ and (5.19) are the required properties for $\rho$ in the proposition. Thus it remains to prove that (5.9), (5.14) and (5.15) imply the existence of $\tilde{\psi} \in C^{1}[0, \infty)$ satisfying (5.16) and (5.17).

Let

$$
\left\{\begin{array}{l}
\psi_{0} \equiv\left\{\psi^{*}+\psi_{*}\right\} / 2 \\
h \equiv \psi_{0}^{\prime} \\
G \equiv \delta F
\end{array}\right.
$$

Then $0 \leq h \leq G$ by (5.14) and (5.15).

First, suppose that

$$
h(r)=G(r) \quad \text { a.e. on }[R, \infty) \text { for some } R>0 .
$$

In this case, for any $\varepsilon>0$, we can take $g \in C[0, \infty)$ such that $g(r)=G(r)$ for $r \geq R, \int_{0}^{R}|h-g|<\varepsilon$ and $0 \leq g \leq G$ (approximate $h$ in $L^{1}(0, R)$ by $g_{1} \in C[0, R]$ with the property $g_{1}(R)=G(R)$ and put $g=\left(g_{1} \vee 0\right) \wedge G$ on $[0, R]$ and $g=G$ on $[R, \infty))$. Let $\tilde{\psi}(r)=\psi_{0}(R)+\int_{R}^{r} g(t) d t$. Then we have by $(5.21)$

$$
\left\{\begin{array}{l}
\tilde{\psi}(r)=\psi_{0}(r) \quad \text { for } \quad r \geq R, \\
\left|\tilde{\psi}(r)-\psi_{0}(r)\right|=\left|\int_{r}^{R}(h(t)-g(t)) d t\right|<\varepsilon \quad \text { for } \quad 0 \leq r \leq R .
\end{array}\right.
$$

Therefore, if we choose $\varepsilon=\frac{1}{4} \min _{0 \leq r \leq R}\left(\psi^{*}(r)-\psi_{*}(r)\right)$, which is positive by (5.9), we have (5.16) and (5.17) by (5.20) and by $\tilde{\psi}^{\prime}=g$.

Next, suppose that (5.21) does not hold. Then there exists a sequence $\left\{R_{k}\right\}_{k=1,2, \ldots}$ such that $R_{1}=0, R_{k} \nearrow \infty$ as $k \rightarrow \infty$ and $\int_{R_{k}}^{R_{k+1}}(G-h)>0$. Apply Proposition 5.1 with $(a, b)=\left(R_{k}, R_{k+1}\right)$ and with $h$ and $G$ in (5.20), which can be easily verified to satisfy the assumptions of Proposition 5.1. Let $\varepsilon_{k}=$ $\frac{1}{4} \min _{R_{k} \leq r \leq R_{k+1}}\left(\psi^{*}(r)-\psi_{*}(r)\right)$, which is positive by (5.9). Then by Proposition 5.1 there exist functions $g_{k} \in C_{0}\left(R_{k}, R_{k+1}\right)$ such that 


$$
\begin{gathered}
0 \leq g_{k} \leq G, \\
\int_{R_{k}}^{R_{k+1}}\left|g_{k}-h\right| \leq \varepsilon_{k}, \\
\int_{R^{k}}^{R_{k+1}} g_{k}=\int_{R_{k}}^{R_{k+1}} h .
\end{gathered}
$$

Define $g=g_{k}$ on $\left[R_{k}, R_{k+1}\right]$ for each $k=1,2, \cdots$. Then $g \in C[0, \infty), 0 \leq g \leq G$ on $[0, \infty)$ by $(5.22)$. Therefore, if we define $\tilde{\psi}(r) \equiv \psi_{0}(0)+\int_{0}^{r} g(s) d s, \tilde{\psi} \in$ $C^{1}[0, \infty)$ and $0 \leq \tilde{\psi}^{\prime} \leq G$, which means (5.17) by (5.20). Moreover, it follows from (5.24) that $\widetilde{\psi}\left(R_{k}\right)=\psi_{0}\left(R_{k}\right)$ for $k=1,2, \cdots$, which with (5.23) imply

$$
\left|\tilde{\psi}(r)-\psi_{0}(r)\right|=\left|\int_{R_{k}}^{r}\left(g_{k}(s)-h(s)\right) d s\right| \leq \varepsilon_{k} \quad \text { for } \quad r \in\left[R_{k}, R_{k+1}\right] .
$$

Hence $\tilde{\psi}$ satisfies (5.16) by (5.20) and by the choice of $\varepsilon_{k}$. This completes the proof of the proposition.

\section{References}

[1] Eastham, M.S.P., Evans, W.D. and Mcleod, J.B., The essential self-adjointness of Schrödinger-type operators, Arch. Rational Mech. Anal., 60 (1976), 185-204.

[2] Ikebe, T. and Kato, T., Uniqueness of the self-adjoint extension of singular elliptic differential operators, Arch. Rational Mech. Anal., 9 (1962), 77-92.

[3] Jörgens, K., Wesentliche selbstadjungiertheit singulärer elliptischer differentialoperatoren zweiter ordnung in $C_{0}^{\infty}(G)$, Math. Scand., 15 (1964), 5-17.

[4] Leinfelder, H., Gauge invariance of Schrödinger operators and related spectral properties, J. Op. Theory, 9 (1983), 163-179.

[5] Leinfelder, H. and Simader, C.G., Schrödinger operators with singular magnetic potentials, Math. Z., 176 (1981), 1-19.

[6] Reed, M. and Simon, B., Methods of Modern Mathematical Physics, Vol. II, Academic Press, New York, 1975.

[7] Titchmarsh, E.C., Eigenfunction Expansions Associated with Second-Order Differential Equations, Part II, Oxford University Press, London, 1958. 